\author{
EUROPEAN ORGANIZATION FOR NUCLEAR RESEARCH \\ CERN - AB Department
}

CERN-AB-2007-035

\title{
BUNCH COMPRESSOR FOR BEAM-BASED ALIGNMENT
}

A. Latina, P. Eliasson ${ }^{1)}$, D. Schulte

CERN, Geneva, Switzerland

\begin{abstract}
Misalignments in the main linac of future linear colliders can lead to significant emittance growth. Beam-based alignment algorithms, such as Dispersion Free Steering (DFS), are necessary to mitigate these effects. We study how to use the Bunch Compressor to create the off-energy beams necessary for DFS and discuss the effectiveness of this method.
\end{abstract}

\footnotetext{
${ }^{1)}$ Uppsala University, Uppsala
}

Presented at

PAC07, 22nd PAC Conference, June 25-29, 2007, Albuquerque, USA

Geneva, Switzerland

August 2007 


\title{
BUNCH COMPRESSOR FOR BEAM-BASED ALIGNMENT*
}

\author{
A. Latina, D. Schulte (CERN, Geneva, Switzerland), P. Eliasson (Uppsala University, Uppsala)
}

\section{Abstract}

Misalignments in the main linac of future linear colliders can lead to significant emittance growth. Beam-based alignment algorithms, such as Dispersion Free Steering (DFS), are necessary to mitigate these effects. We study how to use the bunch compressor to create the off-energy beams necessary to DFS and discuss the effectiveness of this method.

\section{INTRODUCTION}

In future linear colliders, small misalignments of quadrupoles and accelerating structures can lead to significant emittance growth when the beam is transported through the linac. Beam-based alignment techniques are thus required.

The alignment procedure foreseen for CLIC and ILC proceeds in several stages: first, a simple one-to-one correction will be applied to steer the beam into the center of all beam position monitors; then Dispersion Free Steering or Ballistic Alignment will be applied and, finally, RF Alignment and wakefield bumps in CLIC, or Dispersion Bumps in ILC, will be used. In this paper, we focus our attention on Dispersion Free Steering. Dispersion Free Steering attempts to cure the emittance growth and to minimize the dispersion at the same time. In order to do this, one nominal beam and one (or more) test beams with different energies, are sent through the machine; then the correctors are varied until these beams follow a common trajectory, which is a dispersion free orbit. Practically, using $n$ bpms and $m$ test beams, the correctors are varied to minimize the function

$$
M=\sum_{i=1}^{n} y_{0, i}^{2}+\sum_{j=1}^{m} \sum_{i=1}^{n} \omega_{j}\left(y_{j, i}-y_{0, i}+\Delta_{j, i}\right)^{2}
$$

where $y_{j, i}$ is the $i$-th BPM reading for the $j$-th beam; $\omega_{j}$ is the relative weight of the $j$-th test beam with respect to the nominal one and the term $\Delta_{i, j}$ is the target dispersion that must be kept into account when correcting a linac that follows the Earth's curvature (zero for a laser-straight machine). Details about DFS in CLIC and ILC can be found in [2] and [3].

In this paper we discuss the use of the bunch compressor to generate the beams for DFS, for the CLIC and ILC main linacs.

* This work is supported by the Commission of the European Communities under the $6^{\text {th }}$ Framework Programme "Structuring the European Research Area", contract number RIDS-011899.

\section{CASE OF CLIC}

\section{Description}

The required bunch length in the CLIC main linac should be $30 \mu \mathrm{m}$ in order to reduce the dilution effect of transverse wakefields on the vertical emittance. Since at the exit of the damping rings the bunch length is about $3 \mathrm{~mm}$, the required compression factor is about 100, which cannot be obtained by a single stage of compression. For this reason, the CLIC bunch compressor has to operate in two stages, that have been designed to be at $1.98 \mathrm{GeV}$ and at $9 \mathrm{GeV}$ respectively. A transfer line and a turn-around, bringing the beam from the first to the second stage of compression, fix the energy and the beam parameters at the entrance of this second stage. Only the second stage of compression can therefore be used to generate the energy difference, at the entrance of the main linac, that is necessary to DFS. The parameters of the bunch compressor stage 2 are shown in Tab. 1.

\begin{tabular}{|l|l|l|}
\hline RF System & & \\
\hline integrated voltage & $\mathrm{MV}$ & 1009.14 \\
phase & $\mathrm{rad}$ & $k \pi$ \\
frequency & $\mathrm{GHz}$ & 30 \\
\hline Magnetic Chicane & & \\
\hline$R_{56}$ & $\mathrm{~mm}$ & -14 \\
norm $s-E$ correlation & $1 / \mathrm{m}$ & -70.5 \\
\hline
\end{tabular}

Table 1: RF system and magnetic chicane parameters of the CLIC bunch compressor stage 2

\section{Generating the Energy Difference}

The RF system of the CLIC bunch compressor stage 2 accelerates the bunches at phase 0: therefore it introduces a correlation between the longitudinal position and the energy but it does not actually change the energy of the bunches. To change the energy, the test beams must be sent off-phase through the accelerating structures. In this case they can be either accelerated or decelerated, according to the sign of their phase offset. With a \pm 10 degrees phase offset, the energies of the test beams are

$$
\begin{aligned}
& \text { 1. } \Delta \phi_{1}=+10 \mathrm{deg} \Rightarrow E_{1}=8.65 \mathrm{GeV}\left(96 \% E_{0}\right) \text { and } \\
& \text { 2. } \Delta \phi_{2}=-10 \mathrm{deg} \Rightarrow E_{2}=9.35 \mathrm{GeV}\left(104 \% E_{0}\right)
\end{aligned}
$$

respectively. Fig. 1 shows the longitudinal phase-space of such off-phase bunches at the entrance and at the exit of the magnetic chicane. 

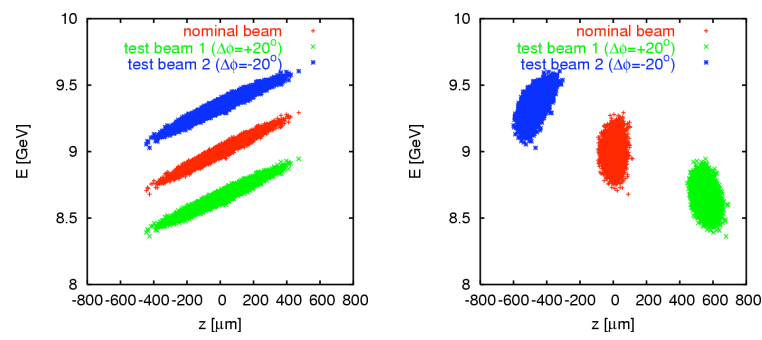

Figure 1: Longitudinal phase space of off-phase bunches at the entrance (left) and at the exit (right) of the magnetic chicane of the CLIC bunch compressor stage 2 .

\section{Simulation Procedure}

The simulations start from a misaligned machine, according to the initial static imperfections listed in Tab. 2 - A nominal beam is sent through the bunch compressor 2 and in the main linac, where the one-to-one correction is applied. Then, the phase of RF structures of the bunch compressor is offset, by 10 and -10 degrees respectively, in order to create two test beams. One should notice that, in doing this, the test beams are shifted longitudinally by the magnetic chicane and are off-phase with respect to the structures in the main linac (see Fig. 1). Before proceeding through the main linac, the beams must be synchronized with the phase of the main linac structures.

To improve the effectiveness of DFS, the test beam starting with a lower energy is accelerated, in the main linac, with a lower gradient than the nominal one, whereas the other test beam starting with a higher energy is accelerated with the same gradient as the nominal beam.

The dispersion free correction is followed by an alignment of the RF structures. Each structures is equipped with an internal beam position monitor. Starting from the upstream end, each structure is moved in order to minimize the average position of the beam in the structure.

Finally, tuning knobs are applied. The knobs consist of a number of accelerating structures that are moved transversely in order to minimize the beam emittance at the end of the linac. In the simulations, the knobs are modeled in a simplified fashion, assuming perfect resolution for the emittance measure. All simulations have been performed using PLACET[1].

\begin{tabular}{|l|l|}
\hline cavity position & $10.0 \mu \mathrm{m}$ \\
cavity pitch & $10.0 \mu \mathrm{rad}$ \\
bpm position & $10.0 \mu \mathrm{m}$ \\
bpm resolution & $100.0 \mathrm{~nm}$ \\
\hline
\end{tabular}

Table 2: Initial static imperfections for the CLIC main linac.

\section{Results}

Fig. 2 compares the results obtained in previous studies[2] (in which we used Gaussian beams created di- rectly at the linac entrance with the desired energy difference, without taking into account the bunch compressor), with the results obtained considering the bunch compressor appropriately. This study confirms the effectiveness of the method, and proposes a way to generate such test beams. We also showed that using realistic beams not only works as expected, but it even further reduced the final emittance growth.

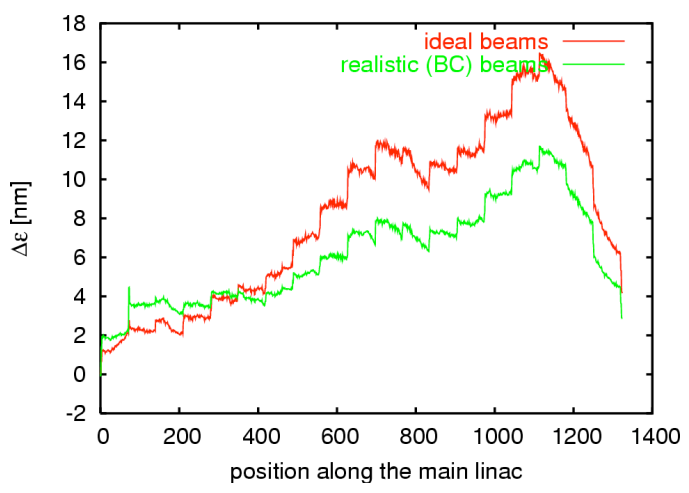

Figure 2: Emittance growth along the CLIC main linac, for a set of ideal, Gaussian, test beams (in red) vs realistic test beams obtained simulating the bunch compressor (in green), after the full alignment procedure.

\section{CASE OF ILC}

\section{Description}

The ILC bunch compressor accelerates the bunches from $4.79 \mathrm{GeV}$ up to $15 \mathrm{GeV}$ and reduces the bunch length from $6 \mathrm{~mm}$ to $300 \mu \mathrm{m}$, before the beam is injected in the main linac. In the ILC bunch compressor, differently from CLIC, the accelerating cavities of the second stage of compression are not at zero phase (but at 22 degrees) and they significantly accelerate the beam. This has a direct impact on the energy of the test beams obtained offsetting the phase of the accelerating cavities. In this case, in fact, the test beams gain a significant energy difference with respect to the nominal one. Fig. 3 shows the energy at the bunch compressor exit as a function of the phase offset.

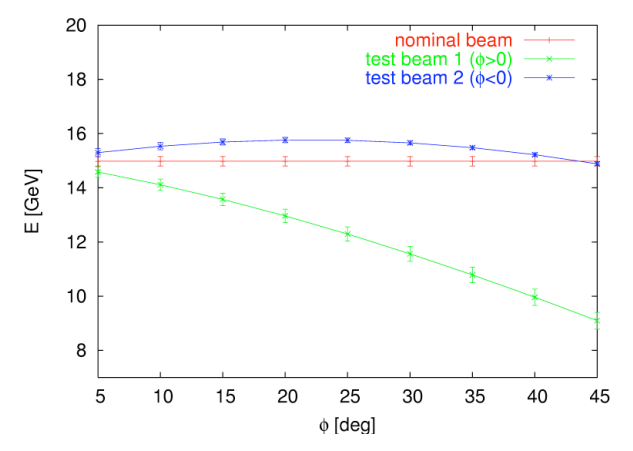

Figure 3: Beam energy at the exit of the ILC BC2 as a function of the phase offset. 


\section{Simulation Procedure and Results}

In our simulations we used the 24 cavity spacing version of the lattice (in which one quadrupole is placed every three cryogenic modules) and analyzed both the cases of a laserstraight linac and of a linac that follows the curvature of the Earth. As phase offset, we used 20 degrees, which leads to the following energies for the test beams:

$$
\begin{aligned}
& \text { 1. } \Delta_{1} \phi=+20 \mathrm{deg} \Rightarrow E_{1} \simeq 80 \% E_{0} \text {, and } \\
& \text { 2. } \Delta_{2} \phi=-20 \mathrm{deg} \Rightarrow E_{2} \simeq 105 \% E_{0} .
\end{aligned}
$$

\begin{tabular}{|l|l|l|}
\hline$\sigma_{\text {quad }}$ & $300 \mu \mathrm{m}$ & quadrupole position \\
$\sigma_{\text {quad roll }}$ & $300 \mu \mathrm{rad}$ & quadrupole roll \\
$\sigma_{\text {cav }}$ & $300 \mu \mathrm{m}$ & cavity position \\
$\sigma_{\text {cav angle }}$ & $300 \mu \mathrm{rad}$ & cavity pitch \\
$\sigma_{\text {bpm }}$ & $300 \mu \mathrm{m}$ & bpm position \\
$\sigma_{\text {res }}$ & $1-2-5-10 \mu \mathrm{m}$ & bpm resolution \\
$a$ & $0-0.2 \%$ & bpm scale error \\
\hline
\end{tabular}

Table 3: Summary of the imperfections in the ILC main linac.

The alignment procedure consisted in the following steps: one-to-one correction, dispersion free steering, then dispersion bumps optimization. All simulations have been performed using PLACET[1].

\section{Case of a laser-straight linac:}

Fig. 4 shows that the final emittance growth obtained after dispersion free steering for with $1 \mu \mathrm{m}$ BPM resolution, and a weight $\omega$ equal to 10000 is well below $2 \mathrm{~nm}$, which is well within the design limits. In this case, Dispersion Bumps are not necessary.

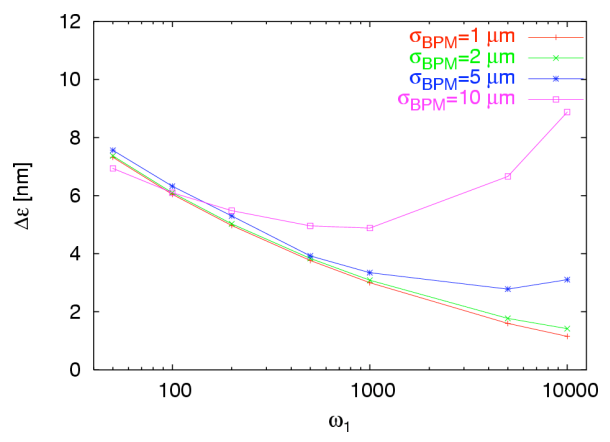

Figure 4: Final emittance growth for an ILC main linac in the laser-straight configuration, as a function of the DFS weights $\omega$, for different BPM resolutions. A final emittance growth of about $2 \mathrm{~nm}$ is well under the design limits.

\section{Case of a linac which follows the Earth's curva-} ture:

Fig. 5 shows the final emittance growth for an ILC main linac that follows the Earth's curvature. Differently from the previous case, in this case DFS is no longer enough to maintain the emittance growth under acceptable limits and further optimization procedures, such as dispersion bumps, are necessary.

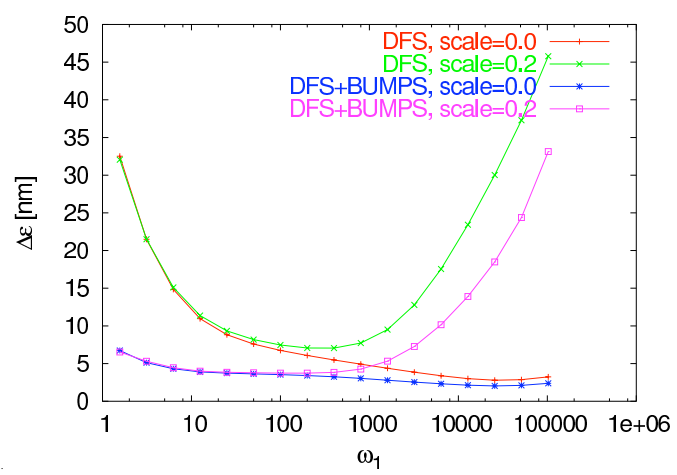

Figure 5: Final emittance growth for an ILC main linac that follows the Earth's curvature, as a function of the DFS weights $\omega$ and in presence of bpm scale errors. Bpm scale errors become relevant for high weights, where DFS is no longer enough to maintain the emittance under acceptable limits and one needs to use further optimization procedures.

\section{CONCLUSIONS}

In this paper we proposed how to use the bunch compressor to align the main linacs of ILC and CLIC, via dispersion free steering. In the case of a laser-straight ILC main linac, the performances of this method lead to excellent results, keeping the final emittance growth to less than $2 \mathrm{~nm}$, which is well below the design limits. In the case of an ILC main linac that follows that Earth's curvature, the bpm scale error showed to be a critical issue and the dispersion steering, although showing good performances, was no longer enough to maintain the emittance under acceptable limits. In this case, further optimization steps such as dispersion bumps must be applied, which however lead to acceptable final emittances, well belows the design limits. In the case of the CLIC main linac, the use of the bunch compressor stage 2 for dispersion free steering, lead to very good results, not only confirming but also improving the results obtained in previous studies.

\section{REFERENCES}

[1] http://savannah.cern.ch/projects/placet

[2] D. Schulte, "Different Options for Dispersion Free Steering in the CLIC Main Linac", EUROTeV-Report-2005-004

[3] P. Eliasson and D. Schulte, "Dispersion Free Steering and Emittance Tuning Bumps in the ILC Linac", EUROTeVReport-2005-021 